\title{
A Mineral Deposit Modelling and Assessment Expert System
}

\author{
Ian Moore \\ Qld. Deptt. of Minerals \& Energy \\ GPO Box 194, Brisbane \\ Australia 4001 \\ imoore@cit.gu.edu.au
}

\author{
Abdul Sattar \\ School of Comput. \& Info. Technology \\ Griffith University, Brisbane \\ Nathan, Australia 4111 \\ sattar@cit.gu.edu.au
}

\begin{abstract}
This paper presents the design and implementation of a knowledge-based system, Queensland Mineral Deposit Expert (QMDE). The primary goal of this system is to assist in the modelling and economic assessment of potential mineral deposits in Queensland. The system produces an assessment of a mineral occurrence's affinity to standard deposits for which economic characteristics such as grade, current production tonnage, employment, and estimated mine value. These parameters are known from the mining operations that have occurred or currently are in progress at these deposits. QMDE is developed using the VPexpert system shell. The system is affordable, can be made easily available on most personal computers, and can be enhanced with minimum effort. It has been successfully implemented in the Queensland mineral environment, and is currently being extended for specific commodity domains such as Gold and Copper. The system can be readily tailored to address specific mineral commodities and environments. The functions are being extended to incorporate intelligent access to large data bases of facts stored on Queensland's mineral deposits through manipulation of data models of mineral occurrence profiles and mining accident statistics.
\end{abstract}

Key words: Expert systems, VP-expert, Mineral Deposits Modelling

\section{Introduction}

An expert system is a software package which emulates the decision-making ability of a human expert in a specific domain. For example, building computer programs for diagnosing a patient based on a given set of symptoms and advising him of an appropriate treatment, the design and diagnosis of faults in electronic circuits, the characterisation of oil and gas fields with an assessment of their hydrocarbon potential or configuration of computer systems (e.g., $[3,4])$. There have been quite a few expert systems developed to assist geologists and mineralogists in exploring potential oil or mineral deposit (e.g., $[2,7,8,9]$ ). One of the problems with the application of a knowledge-based approach to mineral exploration has been the lack of standardised terminology, the lack of consensus among the domain experts on how to describe the field data in different parts of the world using common geologi- cal models and the need to access large fact bases. [4] It is very expensive to import and use an expert system developed somewhere else with different domain settings. Here, our primary motivation is to develop a knowledge-based system which is inexpensive, easily available on personal computers, and able to use a current mineral occurrence descriptive framework in common use within the minerals industry.

We describe the development of a prototype expert system, called Queensland Mineral Deposit Expert (QMDE). Its main goal is to improve the classification and appraisal of Queensland's known and potential mineral deposits. It is required to classify Queensland's mineral deposits to a standard suite of deposit models extracted from known deposits throughout the world with particular emphasis on unique Queensland geological examples. It also appraisals potential mineral occurrences within Queensland against this standard suite to assist in the evaluation of economic viability. The ability to test hypotheses regarding mineralogical, geochemical and lithic profiles against specific Queensland models is desired. Queensland mineral occurrence profiles are stored in the separate database ${ }^{1}$.

We used VP-Expert system shell[1] for the development of QMDE, because it is very inexpensive, easily implemented on IBM PC $^{2}$ which is the most popular personal computer in the industrial environment and has a facility to generate menu select-able unique instances from general entity classes stored in Xbase files. Also, VP-expert offers a simple graphics interface, explanation capability through its "BECAUSE" clause as well as providing facilities to directly access Xbase fact files. Its dual variable facility allows an easy specification of statistically founded knowledge about the mineral deposits. Fact base modification and addition facility provides a learning mechanism of domain semantics through the Xbase structure and modelled frames of economic deposits. The rule base can also be implemented as an updatable class of rule clauses. Consequently individual rules can be modified and new ones added through an externally accessible knowledge base. Another feature utilised in QMDE is the ability to CHAIN several rule

\footnotetext{
${ }^{1}$ All Queensland mineral occurrence profiles and minera production statistics are stored in Xbase files, MINOCC and MPROD,

${ }^{2}$ Trademark of International Business Machines Co.
} 




Figure 1: Logical flow of mineral occurrence settings

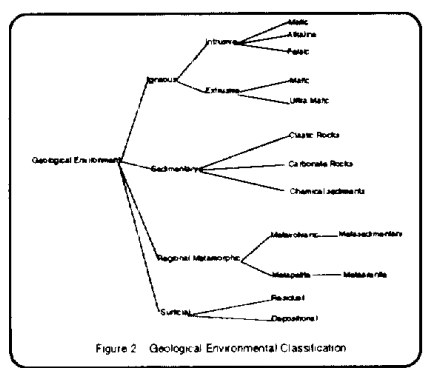

Figure 2: Geological environment classification

bases in a consultation session. Knowledge base chaining has been employed in the development and implementation of the separate QMDE system component explanation interface. The WHILE clause has been employed on some rules so that when the system's WHAT-IF facility is selected by the user during a consultation alternate hypotheses can be investigated.

Next, section gives an overview of the specification of QMDE. Section 3 mentions how a user interacts with QMDE. Section 4 discusses related research work. Finally, we conclude the paper and mention future research directions.

\section{The Specification of QMDE: an overview}

\subsection{Specification of Geological Environ- mental Setting}

The process of specification of a given mineral occurrence setting for evaluation against the modelled deposits is presented in Fig.1. Initially the geological environmental classifier is determined through the specification of the primary, $\mathrm{P}$, and secondary, $\mathrm{S}$, descriptors followed by an third, tertiary, T, descriptor. This hierarchical geological environmental classification is presented in Fig.2.

Following the determination of the mandatory en-

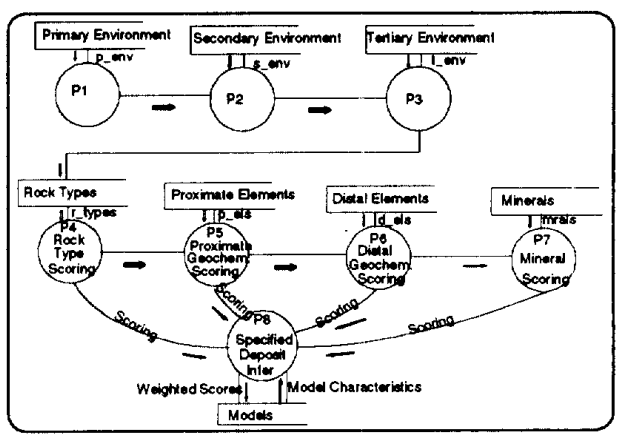

Figure 3: Knowledge Acquisition Cycle

vironmental identifier by the system from the above descriptors, the rock type(s) are specified as dual variables consisting of the rock-type identifier, $R$, and an associated optional probability, $\mathrm{P}$, of occurrence. All identifiers are presented as multiple menu selections. These selections are extracted as unique subclasses within the rock type entity class.

Next optional proximate geochemical elements, G, distal geochemical elements, D, minerals, M, and geological age, A, of the deposit with associated probabilities, $\mathbf{P}$, are specified. The attributes specified at present are the minimal set thought by geologists suitable for the model comparisons.

Fig. 3 sketches the sequence of various types of knowledge acquired about the specific mineral occurrence being evaluated.

\subsection{Specification of Established Deposits}

A NIAM (Nijissen's information Analysis Method) [5] based approach is used for specification of the knowledge of established mineral deposit models and their characteristics accumulated in the various entities in the DB files.

\subsection{Specification of Mineral Production}

A key requirement of the expert system is to tie model inferencing to established current fact base of mineral production of economic deposits represented in the modelled base. Another requirement is to link models to previous production statistics. These data are of prime interest to the local mining industry. Many detailed statistical reports are produced periodically for publication by the Department, The Australian Bureau of Census and Statistics and the Australian Bureau of Agricultural and Resource Economics.

Production statistics for Queensland have been collected since 1983 for fuel minerals (coal, petroleum and liquefied petroleum gas), construction materials, gem-stones and non fuel minerals such as copper, lead, zinc, gold, mineral sands and bauxite operations. At present these data are collected in Xbase standard files which have been made directly addressable from QMDE using the special facility available on VP-expert system shell. 


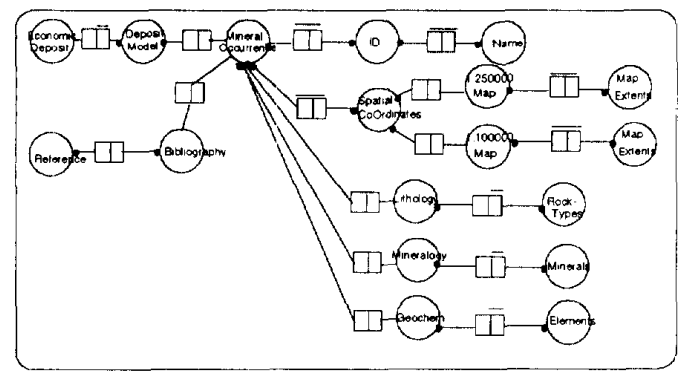

Figure 4: Mineral occurrence profile

\subsection{Specification of Mineral Occurrences}

Another requirement of the system is to eventually allow the linkage to a mineral occurrence data base which contains details of currently known occurrences whose economic viability needs to be assessed. It is from this base that the affinity to the standardised model frames recognised by QMDE are scored in terms of their geological affinity to the standardised model frames The NIAM data model for the MINOCC class of facts is detailed in Fig.4. This diagram depicts only those entities of the MINOCC system presently accommodated by the QMDE system.

\subsection{Model Inference}

QMDE employs a scoring system for weighting the affinity of the given occurrence's geological characteristics to the established model set. For each attribute specified an optional probability or certainty of occurrence can be declared. Upon completion of the specification of a class of geological attributes such as rock-types the associated dual variables are sorted in descending order of certainty. This weighting is then accumulated in the score attribute attached to each standard model.

At present no priority of importance has been attached to a particular geological attribute class over another. This could be easily accommodated however with further employment of Bayesian probabilities. There is an implied priority in that the declaration of items within a geological class is the decision exclusively of the user. The resultant model selection is that with the highest score. The user may than optionally view Australian examples displayed on a state basis mining operations.

\section{User Interaction}

The user interacts with the system through a series of windows offering multiple selections. Depositional geological environmental rock type, minerals, proximal and distal geochemical elements and inferred geological age are displayed on screen, as illustrated in Fig.6.

Our system QMDE has the facility to extract unique subclasses and subclass instances from an entity class such as minerals and rock-types. The sys-

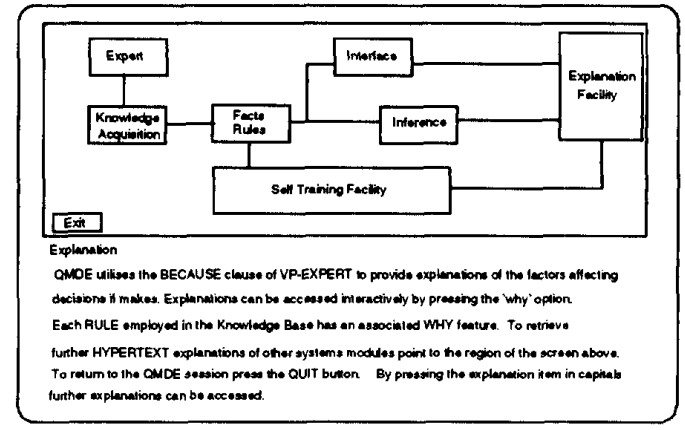

Figure 5: User information window

tem associates a $d u a l$ variable to each unique instance. The uncertainties are specified at the menu selection through pressing the Home key and entering an uncertainty factor as an integer in the range 0 to $100 .^{3}$

The knowledge base in QMDE consists of rules represented by using if-then-else-because constructs of VP-expert. These rules can be fired in a backward chaining inference method. Also, 'daemons' can be fired as specified in a forward chaining approach through the Whenever rule. The ability to chain one knowledge base to other in a structured integrated manner, has been employed in this system to permit access to a help facility. This help module is presented as a schematic (as shown in Fig.5) incorporating hot-regions attached to the sections of the diagram to which the user points with an attached mouse or function keys.

Associated with each hot-region, accessed through whenever rule, are hyper-text information segments. QMDE permits interaction with diagrams defined as pixel coordinates and employs a separate drawing software tool such DRAW PERFECT to define the diagram. This facility could be used to generate an HPGL formatted file. An interface module was then written to convert this HPGL file to screen coordinates.

\section{Related Work}

Like the existing mineral deposit exploration expert systems (e.g., Prospector [7] and mu-Prospector [9]), QMDE has the similar functionality in term of classifying unknown deposits against standardised models. However, QMDE is significantly different from the previously developed systems, specially Prospector and mu-Prospector.

Prospector and muPropsector employ semantic nets and frame based techniques to represent the mineral domains; use only limited number of deposit models (five in case of Prospector); use forwarding chaining as main inference method; have textual user consultation with primitive screen layout; rely on an a prior specification of the statistical analysis of the major

\footnotetext{
${ }^{3} \mathrm{~A}$ detailed account of experimental results of QMDE will be presented in the larger version of this paper [6]
} 




Figure 6: An example of user interaction

characteristics of the domain; and required extensive cost and time resources.

QMDE employs semantic nets and frames as knowledge representation schemes, but they allow the expert to incrementally update the domain knowledge; currently uses over fifty models; uses combination of forward and backward chaining as inference strategy; provides graphics window based user interface with online help and explanation facilities; has ability to link external databases, that allows us to use the large amount of mineral occurrence profiles accumulated in Queensland over the years without actually re-representing the existing domain knowledge; and allows the knowledge engineer to dynamically update the probability assignments. QMDE was inexpensive to develop both from the time and cost view points.

Nevertheless our system takes advantages of most relevant features of the previously developed systems and the evolving mineral deposit modelling methodology within mineral exploration. Also, it provides an improved operational expert system within Australian mineral context.

\section{Conclusion}

We have presented an overview of QMDE, Queensland Mineral Deposit Expert system. The system produces an assessment of a mineral occurrence's affinity to standard deposits for which economic characteristics such as grade, current production tonnage, employment, and estimated mine value. These parameters are known from the mining operations that have occurred or currently are in progress at these deposits. Our approach has been very successful with respect Queensland mineral environment. A comparison with the previously developed expert systems suggests that QMDE takes the advantages of the recent advances in the mineral deposits methodology. The application of expert systems technology is vital to an economy such as Australia's being so heavily dependent on the mineral resource sector in particular and the primary resource sector in general for much of its export income. It is believed that knowledge based technology can play a significant role in new mineral exploration and resource assessment and intelligent query to mineral databases. The system is currently being extended for specific commodity domains such as Gold and Copper.

\section{Acknowledgement}

The first author likes to acknowledge the resources and financial assistance provided by the Queensland Department of Minerals and Energy. We like to thank Fredarick Bruvel for assistance. Also, we would like to thank Richard Hagen for his useful comments.

\section{References}

[1] VPExpert: Rule based expert system development tool. User manual, Paperback Software Intl., 1989.

[2] J.D. Bliss. Developments in Mineral Deposit Modelling. United States Geological Survey 2004, 1992.

[3] B. G. Buchanan and E. H. Shortliffe, Rule-Based Expert Systems. Addison Wesley, Reading, MA, 1984

[4] Cheong D.K., Strobel J., Biswas G., Lee G., Kendall C.G., Cannon R., and Bezdek J. Playmaker, a knowledge based expert system. Geobyte, $7(6): 28-41,1993$.

[5] Nijssen G.M and Halpin T.A. Conceptual Schema and Relational Data Base Design. Prentice Hall, 1989.

[6] I. Moore and A. Sattar. QMDE: A Mineral Deposit Modelling and Assessment Expert System. Research report (forthcoming), School of Computing and Information Technology, Griffith University, Nathan, Brisbane, Australia, 1993.

[7] Duda R. and R. Reboh. AI and Decision Making: The PROSPECTOR Experience. Norwood, N.J., 1984.

[8] Lister R, Ali K., Buda R., Horsfall C., and Buntine W. GOLD: an expert system for mineral identification from reflectance spectra. Technical report, Basser Department of Computer Science, University of Sydney, 1987.

[9] McCammon R.B. The MuProspector mineral consultant system. Bulletin 1697, United States Geological Survey, 1986. 